\title{
Evaluation of computer-based laboratory simulation models to teach scientific research strategies
}

\author{
GERALD R. KISSLER \\ Office of the Chancellor, University of California, Los Angeles, Los Angeles, California 90024
}

\begin{abstract}
The LABSIM simulation program is described, followed by a general evaluation of simulation models and an indication of possible ways to improve the effectiveness of simulation models.
\end{abstract}

This paper has three major purposes: (1) to describe the simulation program developed at Washington State University, (2) to review attempts to evaluate the effectiveness of laboratory simulation models, and (3) to offer some suggestions on ways to improve the effectiveness of simulation models. The simulation program developed at Washington State University grew out of my interest in the use of computers to improve instruction. My interest shifted from computer-assisted instruction (CAI) to using computer programs instead of calculators to teach statistics when Johnson (1971) described the DATACALL game at the Conference on Computers in the Undergraduate Curricula. It seemed that something like the DATACALL game could be useful in teaching the principles of experimental design.

The computer program developed at Washington State University in the fall of 1971 , called LABSIM, ${ }^{1}$ was similar to the DATACALL game. Unlike the DATACALL program, LABSIM was written in PL/I, and the model itself is a general-purpose multiple regression equation of the form:

$$
\begin{aligned}
\mathrm{DV}= & \mathrm{B}_{1} \times I \mathrm{IV}_{1}+\mathrm{B}_{2} \times I V_{2} \cdots \mathrm{B}_{\mathrm{n}} \times \mathrm{IV}_{\mathrm{n}} \\
& +\mathrm{B}_{\mathrm{n}+1} \times \mathrm{IV}_{1} \times \mathrm{IV}_{2} \cdots+\mathrm{e}
\end{aligned}
$$

where DV is the dependent variable, $B_{1}$ is the beta weight associated with the first independent variable, $\mathrm{IV}_{1}$ is the first independent variable, and $\mathrm{e}$ is an error term produced by a random number generator which gives variability to the scores for different Ss.

The program was written around this general-purpose multiple regression equation so that new models for simulating different experiments could be developed by changing the input data cards and without modifying the computer program. This was important because many of those who might be interested in using LABSIM or developing a model would know little or nothing about computer programming. This simplified format led to the development of the following models: (1) Knowledge of the correct response-Effect of feedback in programmed instruction; (2) Communication networks-Social psychology;
(3) Desensitization-Clinical Psychology; (4) Behavior modification-Classroom management; (5) Decision times for alphabetic order-Cognitive processes; (6) Spacing of practice-Motor learning; (7) Adaptation level and judgment of line length-Psychophysics; (8) Concept formation and concept shifts-Developmental psychology; and (9) Serial position effects in free recall-Verbal learning.

The LABSIM program was written in both an on-line and a batch version. Because of the particular interactive programming system evailable, the on-line version was limited to experiments with only two groups of Ss. After signing on to the interactive program, the student was asked a number of questions about the experiment he wanted to design. The student entered the number of Ss to be run in each group and his choices for the values of each of the independent variables. The program then printed the value of the dependent variable for each $S$ in both groups and some summary statistics, such as means, standard deviations, and a t test for the difference between means.

The batch version of the program ran in only a few seconds, but due to procedures at the Computing Center, it usually took $1.3 \mathrm{~h}$ before a student's output would be available. Without the core restrictions imposed by the interactive system, the batch version could simulate more complex experimental designs, including parametric, factorial, and ABA designs.

LABSIM provides the instructor with flexibility in the type and amount of information generated. It can handle discrete, continuous, and range variables. Subroutines are provided so that the model can sample from a rectangular or normal distribution, or any other form of cumulative distribution established by the instructor. LABSIM can print or suppress summary statistics or error messages which indicate the problems in the student's experimental design. The program can calculate the cost of an experiment in points if the instructor wishes to use it as a game the way Johnson used DATACALL. LABSIM also automatically checks the student's choice for each independent variable and the predicted value of the dependent variable to insure that they are within the valid ranges specified by the instructor. 


\section{EVALUATION OF SIMULATION MODELS}

Simulation models and games have been developed and tested in a number of disciplines, including business, economics, genetics, and chemistry. In addition, each of the participants in this symposium has tried to evaluate the model he developed to simulate experiments in psychology. The results of these attempts at evaluation can be summarized into four basic findings.

First, simulation models often save time over traditional laboratory instruction, but seldom have significant differences in achievement been reported. While the saving in time is an important finding consistent with intuition, the failure to find significant differences in achievement has been disappointing. These failures could be attributable to response measures which were not sensitive enough to detect real differences in learning. For example, our multiple-choice tests were too short to be reliable, and we encountered reliability problems in grading essay questions. The failure to find significant differences in achievement might also be attributed to the fact that we have had more experience with traditional laboratory instruction. Students might have to learn how to learn from simulation models, and instructors might have to learn how to use these models effectively. This is consistent with Anderson and Faust's (1973) comment that wo should be sure that a new instructional technique does what it is supposed to do before we compare it to traditional forms of instruction which have been revised and improved over a long period of time. Further support for this thesis comes from recent reports of significant differences in achievement with simulation models that have been used for several terms.

Second, it has often been reported that the use of simulation models leads to increased student interest. However, we did not find differences in student interest between real and simulated experiments on an attitude survey. We did find that students initially liked experiments run with the on-line version better than the batch version. This initial superiority of on-line simulation may have been due to the novelty of using remote terminals.

Third, the reduction in time associated with using the computer can save money. For example, during one semester, the course in experimental methods was taught without teaching assistants. Instead of running the experiment for $3 \mathrm{~h}$ in the laboratory, students were given a brief demonstration of the apparatus and procedure, and then used the computer to simulate the experiment. This procedure reduced the cost for a class of 25 students from $\$ 180$ per experiment in the laboratory, to $\$ 80$ for on-line simulation and $\$ 2$ for batch simulation.

Fourth, some experiments have been simulated which could not have been done in the laboratory. For example, it would not have been possible to allow undergraduate students to use real clients to run an experiment comparing desensitization to psychotherapy. Instead of real clients, the students used LABSIM to simulate the results of such an experiment, after they had been shown a video-taped demonstration of the desensitization process. A similar technique was used to introduce students to $\mathrm{ABA}$ designs as they are used to investigate the effect of behavior modification techniques on the control of disruptive classroom behavior. Both the desensitization and behavior modification simulated experiments were effective in teaching valuable principles, but they could not have been conducted in the laboratory.

\section{POSSIBLE WAYS TO IMPROVE THE EFFECTIVENESS OF SIMULATION MODELS}

Several techniques have been suggested to improve the effectiveness of simulation models. One technique is to allow students to build their own models. Main (1972) has encouraged graduate students at the University of Michigan to build models around their thesis or dissertation data. This increases the number of models available to an instructor, and it teaches the graduate student model building. Alternately, one could let undergraduate students in the class run the experiment in the laboratory, and then use the data collected to build a model. This technique could be used in a class on statistics or model building, and could form the basis for a class that would integrate statistics and experimental design.

Another technique for improving the effectiveness of a simulation is to add points to make it a game. Johnson's DATACALL was a game because students were charged points for every $S$ run and every independent variable controlled. They received points by "publishing" the results of their experiments. This type of game environment emphasizes the costs and payoffs associated with research in the "real world." The points accumulated at the end of the game could be used to determine the student's grade. One could raise the level of competition in the game if the computer were also allowed to play the game, or through some form of intercollegiate competition, as has been done with business games (Holmes, 1972).

Simulation is probably most effective when combined with other types of instruction. A combination of laboratory and simulation experiences would seem to be the most effective way to insure that students learn both data gathering skills and the principles of experimental design. Small group discussion can be used to review and emphasize the effects of different types of experimental designs. We tried to combine simulation with the feedback normally associated with computer-assisted instruction (CAI) by programming error messages which tell the student if he makes errors in his experimental design. By using the storage and retrieval capabilities of the computer, students could learn to use standard 
statistical programs to analyze a data base generated by a simulation model. Further experimentation will help us to determine the most effective way to integrate simulation into the psychology curriculum.

\section{REFERENCES}

Anderson, R. C., \& Faust, G. W. Educational psychology: The science of instruction and learning. New York: Dodd, Mead, 1973.

Holmes, J. D. S. New approaches to business simulations. In Proceedings of the 1972 conference on computers in the undergraduate curricula. Atlanta, Georgia: Southern Regional Education Board, 1972.

Johnson, R. DATACALL: A computer-based simulation game for teaching strategy in scientific research. In Proceedings of the 1971 conference on computers in the undergraduate curricula. Hanover, New Hampshire: Computer-oriented Materials for Undergraduate Teaching, 1971.

Main, D. Toward a future-oriented curriculum, American Psychologist, 1972, 27, 245-248.

\title{
Teaching research theory and application through on-line simulation
}

\author{
ARTHUR CROMER \\ University of Louisville, Louisville, Kentucky 40208
}

The development of the University of Louisville program for teaching experimental psychology and statistics through computer simulation techniques is described.

In the spring of 1971 , we began to replace the required undergraduate course in experimental psychology, which emphasized the theory of research, with a course that emphasized practice as well as theory. For purposes of education, we felt it necessary to provide a student with the opportunity for repeating an experiment many times with minor or even major changes. Most experiments that can be repeated easily are too simple to provide meaningful experience to the student, so the departmental computer was used to simulate the data collection portion of the experiment. The student was still responsible for reading the literature, seeking out meaningful research questions, planning the experiments, analyzing, and reporting the results. By combining these simulations with live experiments, where the primary emphasis was on the techniques necessary for running the experiments and collecting the data, a complete balanced coverage was provided for the entire range of research training.

This technique has been used successfully in about 22 sections (20 students each) of the experimental psychology course offered since the fall of 1971 at the University of Louisville, and at several other colleges and universities (Johnson, 1971; Main \& Head, 1971; Thurmond \& Cromer, 1972; Cromer, 1973; Hallenbeck \& Welch, 1974; Kissler, 1974; Stout, 1974). The students can learn to see not only questions that can be answered by a single experiment, but questions requiring a series of experiments encompassing an entire research program in order to take advantage of results obtained along the way. Some of the experimental models used in these classes have as few as 2 independent variables and 1 dependent variable, while others have 6 to 12 independent and 2 to 4 dependent variables. For example, in one simulation, there are 58,320 possible experiments that could be conducted; this does not include varying the number of Ss. This puts the student in a situation where he must formulate hypotheses in order to obtain results. With most of the computer models employed, the student would be completely lost without some idea of how to proceed intelligently.

It became evident that in order to teach this course effectively, the student must have a background in statistics. At the same time, the problems of motivating the nonquantitative students in sophomore statistics courses using only examples from a book had to be handled. Last year, a series of 22 programs covering the areas of general statistics, probability, correlation, and regression, nonparametrics, distributions, and analy sis of variance were developed to be used in teaching statistics. Some are intended as demonstrations, but most are intended to be used by the students in an on-line situation to aid in answering questions not otherwise answerable. For example, "For the two variables $\mathrm{X}$ and $Y$, given the following four pairs of numbers, choose another pair so that the two variables have a correlation of $-.50 \pm .02 . "$ Only when a student can instantly obtain a correlation can such a question be realistically 